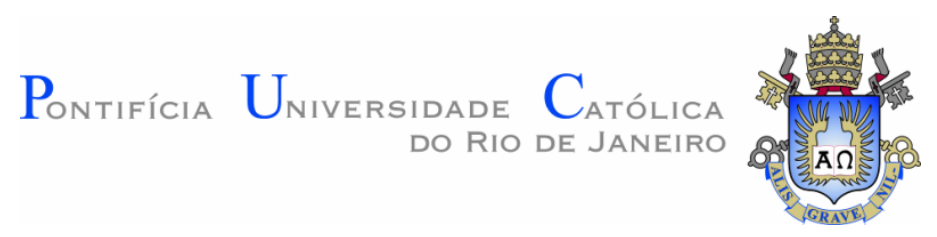

Leandro Fernandes Guimarães

Um Framework para Desenvolvimento de Agentes Autoadaptativos em Dispositivos Móveis

Dissertação de Mestrado

Dissertação apresentada como requisito parcial para obtenção do título de Mestre pelo Programa de PósGraduação em Informática da PUC-Rio.

Orientador: Prof. Carlos José Pereira de Lucena

Rio de Janeiro

Abril de 2012 


\title{
Um Framework para Desenvolvimento de Agentes Autoadaptativos em Dispositivos Móveis
}

\begin{abstract}
Dissertação apresentada como requisito parcial para obtenção do título de Mestre pelo Programa de PósGraduação em Informática da PUC-Rio. Aprovada pela Comissão Examinadora abaixo assinada.
\end{abstract}

Prof. Carlos José Pereira de Lucena

Orientador

Departamento de Informática - PUC-Rio

Prof. Hugo Fuks

Departamento de Informática - PUC-Rio

Prof. Firmo Freire

Departamento de Informática - PUC-Rio

Prof. Soeli Teresinha Fiorini Departamento de Informática - PUC-Rio

Prof. José Eugênio Leal Coordenador(a) Setorial do Centro Técnico Científico - PUC-Rio 
Todos os direitos reservados. É proibida a reprodução total ou parcial do trabalho sem autorização da universidade, do autor e do orientador.

\section{Leandro Fernandes Guimarães}

Graduou-se como Bacharel em Ciência da Computação pela Universidade Federal de Viçosa em 2010. Durante o Mestrado foi bolsista CAPES e desenvolveu um trabalho aplicado em análise de risco geoambiental para dispositivos móveis. Atualmente é analista de riscos de negócio parametrizados por sistemas integrados de gestão na Ernst \& Young Assessoria Empresaria Ltda.

Ficha Catalográfica

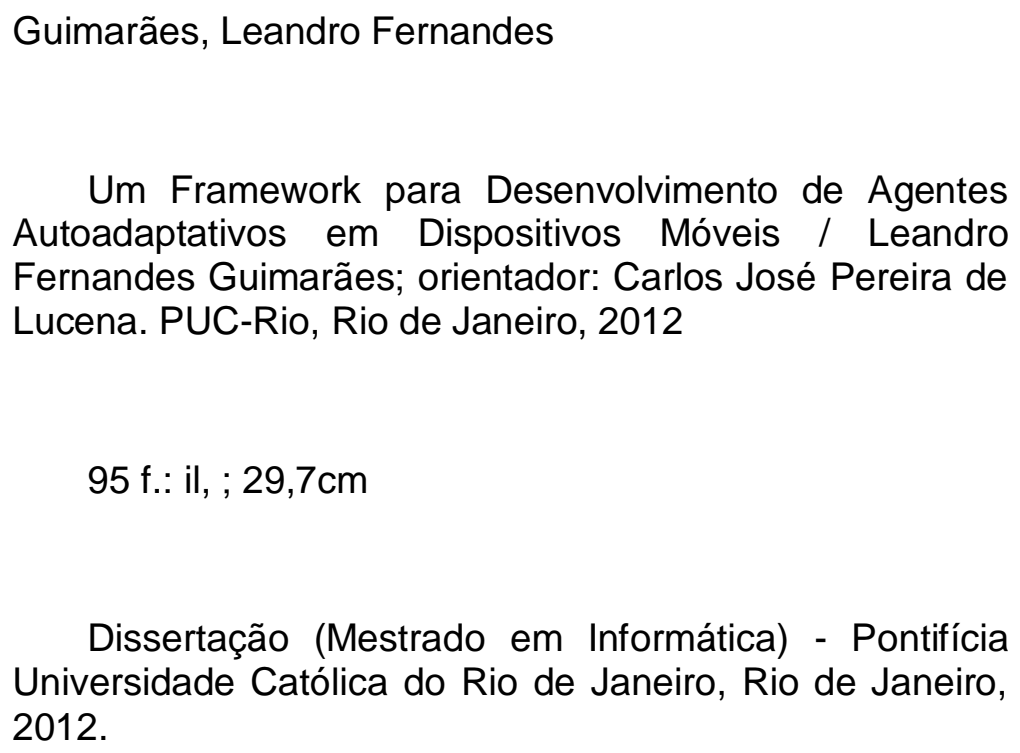

Um Framework para Desenvolvimento de Agentes Autoadaptativos em Dispositivos Móveis / Leandro Fernandes Guimarães; orientador: Carlos José Pereira de Lucena. PUC-Rio, Rio de Janeiro, 2012

95 f.: il, ; 29,7cm

Dissertação (Mestrado em Informática) - Pontifícia Universidade Católica do Rio de Janeiro, Rio de Janeiro, 2012.

Inclui referências bibliográficas.

1. Informática - Teses. 2. Computação Móvel. 3. Serviços Web. 4. Computação Autonômica. 5. Autoadaptação. 6. Sistemas Multiagentes. 7. Framework. I. Lucena, Carlos José Pereira de. II. Pontifícia Universidade Católica do Rio de Janeiro. Departamento de Informática. III. Título. 


\section{Agradecimentos}

Ao meu orientador pelo pela paciência e inestimável apoio prestados durante a realização deste trabalho e, principalmente, pela oportunidade de muito aprender com a experiência e conhecimento científico que lhes pertence.

Aos meus pais por sempre acreditar e respeitar minhas decisões e por nunca deixar que as dificuldades superassem meus sonhos. Aos meus irmão, amigos e namorada por pelo amor, amizade, e apoio depositados, além da companhia por todos esses anos.

Aos meus colegas da PUC-Rio, por tornarem o ambiente de pesquisa mais alegre e descontraído além de toda colaboração durante o trabalho. Em especial para os amigos Baldoino, Carlos, Dárlinton, Elder, Marx e Sérgio.

À CAPES e à PUC-Rio, pelos auxílios concedidos, sem os quais este trabalho não poderia ter sido realizado. 


\section{Resumo}

Guimarães, Leandro Fernandes; Lucena, Carlos José Pereira de. Um Framework para Desenvolvimento de Agentes Autoadaptativos em Dispositivos Móveis. Rio de Janeiro, 2012. 95p. Dissertação de Mestrado Departamento de Informática, Pontifícia Universidade Católica do Rio de Janeiro.

O progresso tecnológico da computação móvel associada à Internet promove a concretização de uma visão futurista em que os usuários acessam dados e serviços a qualquer momento e em qualquer lugar. Entretanto, esta visão expõe questões importantes no desenvolvimento de aplicações, pois se deve dar maior atenção para a comunicação entre dispositivos móveis e serviços web, considerando restrições de processamento, armazenamento de dados, diversidade de padrões e disponibilidade dos serviços. Esta dissertação explora aspectos de engenharia de software como computação autonômica, frameworks e sistemas multiagentes no desenvolvimento de aplicações para dispositivos móveis dando ênfase ao gerenciamento do uso de serviços web. Para consolidar os conceitos abordados e prover um guia que facilite o desenvolvimento de aplicações autoadaptáveis, propõe-se um framework para auxiliar o desenvolvimento de aplicações para computação móvel. Para ilustrar a utilização do framework são apresentadas duas aplicações. A primeira aplicação é um sistema que apresenta o risco de deslizamento de massa em uma área a partir do cálculo de susceptibilidade baseado em informações sobre clima, relevo e solo. A segunda aplicação faz parte de um sistema de agência de viagens que define um pacote de viagens que melhor atende às necessidades do usuário.

\section{Palavras-chave}

Computação Móvel; Serviços Web; Computação Autonômica; Autoadaptação; Sistemas Multiagentes; Framework; Dispositivos Móveis. 


\section{Abstract}

Guimarães, Leandro Fernandes; Lucena, Carlos José Pereira de (Advisor). A Framework for Developing Self-Adaptive Agents in Mobile Devices. Rio de Janeiro, 2012. 95p. MSc Dissertation - Departamento de Informática, Pontifícia Universidade Católica do Rio de Janeiro.

Technological progress in mobile computing and the Internet promotes an achievement of the futuristic vision in which users have access to data and services anytime and anywhere. However, this vision realization brings important issues to application development, because more attention is required to the communication between mobile devices and web services, especially regarding processing and data storage constraints, diversity standards and availability of services. This dissertation explores aspects of software engineering as autonomic computing, multi-agent systems and frameworks in developing applications for mobile devices, emphasizing autonomic management of web services use. Aiming the consolidation of the investigated concepts and provide a guide to facilitate the development of self-adapting applications, a framework is proposed to help in applications development for mobile computing. The development of two applications illustrates the framework appliance. The first application is a system that presents the risk of mass sliding in an area through a susceptibility calculation based on information of climate, topography and soil. The second application is part of a travel agency system that defines a travel package that best fit user needs.

\section{Keywords}

Mobile Computing; Web Services; Autonomic Computing; Self-Adaptation; Multi-agent Systems; Framework; Mobile Devices. 


\section{Sumário}

1 Introdução 11

1.1. Limitações das Abordagens Atuais 12

1.2. Solução Proposta e Principais Contribuições 13

1.3. Organização do Documento 14

2 Fundamentos Teóricos 15

2.1. Serviços Web 16

2.2. Computação Móvel 18

2.3. Computação Autonômica 21

$\begin{array}{ll}\text { 2.4. Sistemas Multiagentes } & 24\end{array}$

3 Framework 32

3.1. Diagrama de Classes $\quad 34$

3.2. Pontos Fixos $\quad 49$

3.3. Pontos Flexíveis 49

4 Aplicações $\quad 51$

4.1. Escorregamento de Massas 51

4.2. Agência de Viagens 65

5 Trabalhos Relacionados $\quad 79$

6 Conclusões e Trabalhos Futuros $\quad 87$

$\begin{array}{lr}\text { Referências Bibliográficas } & 91\end{array}$ 


\section{Lista de figuras}

2.1 - llustração simplificada da arquitetura dos serviços web.

2.2 - Arquitetura do sistema operacional Android (GOOGLE, 2010b). 20

2.3 - Atributos da computação autonômica (MILLER, 2005). 23

2.4 - Máquina de estados dos agentes FIPA (FIPA, 2002). 26

2.5 - llustração da execução no modo Stand-alone. 28

2.6 - llustração da execução no modo Split. 28

2.7 - Ilustração simplificada da plataforma do agente (FIPA, 2002). 29

2.8 - llustração da hierarquia de comportamentos de um agente. 31

3.1 - llustração do Control loop elaborado pela IBM (BELL, 2004). 33

3.2 - Visão geral do framework proposto. 35

3.3 - Atividade Monitor. 36

3.4 - Atividade Analyze. 38

3.5 - Atividade Plan. $\quad 41$

3.6 - Fragmento da árvore de similaridade. 42

3.7 - llustração de solicitação via padrão FIPA ACL. 43

3.8 - llustração de resposta via padrão FIPA ACL. 43

3.9 - Similaridade entre o Problema 1 e o Caso 1.

3.10 - Atividade Execute. 45

3.11 - llustração da atividade do agente JadeGatewayAgent. 46

3.12 - llustração da tradução de uma mensagem ACL para SOAP. $\quad 47$

3.13 - llustração da tradução de uma mensagem SOAP para ACL. $\quad 47$

3.14 - WSDL do novo serviço. 48

3.15 - llustração dos pontos fixos e flexíveis do framework. 50

4.1 - Atividade Monitor de um ACR. 53

4.2 - Atividade Analyze de um ACR. 54

4.3 - WSDL da Solução do Caso 1.

4.4 - WSDL da Solução do Caso 2.

4.5 - Atividade Plan de um ACR. 58 
4.6 - Ilustração do descritor do serviço prestado pela Solução 1.60

4.7 - Ilustração do descritor do serviço prestado pela Solução 2.61

4.8 - Ilustração da similaridade entre o Problema Atual e a Solução 1. 62

4.9 - llustração da similaridade entre o Problema Atual e a Solução 2. 63

4.10 - llustração da transcrição da mensagem ACL para SOAP. $\quad 64$

4.11 - llustração da transcrição da mensagem SOAP para ACL. $\quad 64$

4.12 - Atividade Monitor de um AAV. 67

4.13 - Atividade Analyze de um AAV. 68

4.14 - WSDL da Solução do Caso 1.

4.15 - Descritor DF da Solução do Caso 2.

4.16 - Atividade Plan de um AAV. 73

4.17 - llustração do descritor do serviço prestado pela Solução $1 . \quad 75$

4.18 - llustração do descritor do serviço prestado pela Solução $2 . \quad 76$

4.19 - Ilustração da similaridade entre o Problema Atual e a Solução 1. 77

4.20 - Ilustração da similaridade entre o Problema Atual e a Solução 2. 78 
Se há pessoas que não estudam ou que, se estudam, não aproveitam, elas que não se desencorajem e não desistam; se há pessoas que não interrogam os homens instruídos para esclarecer as suas dúvidas ou o que ignoram, ou que, mesmo interrogando-os, não conseguem ficar mais instruídas, elas que não se desencorajem e não desistam; se há pessoas que não meditam ou que, mesmo que meditem, não conseguem adquirir um conhecimento claro do princípio do bem, elas que não se desencorajem e não desistam; se há pessoas que não distinguem o bem do mal ou que, mesmo que distingam, não têm uma percepção clara e nítida, elas que não se desencorajem e não desistam; se há pessoas que não praticam o bem ou que, mesmo que o pratiquem, não podem aplicar nisso todas as suas forças, elas que não se desencorajem e não desistam; o que outros fariam numa só vez, elas o farão em dez, o que outros fariam em cem vezes, elas o farão em mil, porque aquele que seguir verdadeiramente esta regra da perseverança, por mais ignorante que seja, tornar-se-á uma pessoa esclarecida, por mais fraco que seja, tornar-se-á necessariamente forte.

Confúcio, 'A Sabedoria de Confúcio' 